\title{
Aseptic intussuscepted incidental appendectomy: three successful cases
}

\author{
Evangelia Rachmani, Zacharias Zachariou, Ulf Kessler \\ Berne, Switzerland
}

Background: Incidental appendectomy remains a controversial issue. We aimed to collect experience using a modified surgical technique that could be applied securely in infants.

Methods: We performed aseptic intussuscepted incidental appendectomy (AIIA) in three patients using a technique that is thought to assure appendix necrosis along with intact cecal wall.

Results: There was no perioperative morbidity due to AIIA in the three patients. In two infants the necrotic appendix was found in the diaper. One infant died secondary to diaphragmatic hernia. Autopsy with histological examination revealed that the cecum was intact along with appendix necrosis.

Conclusions: Modified AIIA could securely be performed in the 3 reported cases. We advocate prospective evaluation of the method.

World J Pediatr 2013;9(4):369-372

Key words: appendectomy; appendicitis; inversion appendectomy

\section{Introduction}

$\mathrm{H}$ istorically the argument for performing incidental appendectomy was the fact that the abdomen was already open, and the removal of the appendix would prevent a later emergency operation for acute appendicitis. In addition, the inversion of the

Author Affiliations: Department of Pediatric Surgery, Inselspital, University of Berne, Berne, Switzerland (Rachmani E, Kessler U); University of Berne, Berne, Switzerland (Zachariou Z)

Corresponding Author: Ulf Kessler, MD, Department of Pediatric Surgery, University of Berne, Inselspital, CH-3010 Berne, Switzerland (Tel: 00413163221 11; Fax: 00413163292 92; Email: ulf.kessler@ insel.ch)

doi: 10.1007/s12519-013-0422-4

(C)Children's Hospital, Zhejiang University School of Medicine, China and Springer-Verlag Berlin Heidelberg 2013. All rights reserved. appendix into the cecal lumen might prevent peritoneal contamination. However, the chance of missing appendicitis has become low in the era of managed care, ultrasonography, MRI-imaging and laparoscopy. Above that, several complications as bleeding or intussusception were reported following inversion appendectomy. ${ }^{[1-4]}$ Finally, according to the frequency of associated malformations in candidate newborns for incidental appendectomy, the appendix might be precious for a stoma to the bowel or other hollow organ during later life. ${ }^{[5]}$

However, incidental appendectomy might be considered in special circumstances. In particular, when a high rate of complications in an abdominal relaparotomy during later life might be expected as for example in gastroschisis closure, malrotation, or others. Here abdominal contamination could be minimized by aseptic intussuscepted incidental appendectomy (AIIA). Since the reported complications after AIIA were mainly due to incomplete necrosis of the appendix, leaving a large appendiceal stump leading to bleading or cecocolic intussusception, ${ }^{[1-4]}$ we hypothesized, that the application of a meticulous surgical technique in selected patients might be justified.

\section{Methods}

In a 2-year period (2008, 2009), we performed AIIA in three infants. The parents gave their informed consent for anonymous publication.

Fig. 1 shows the surgical steps, which were equally applied in all three patients: the appendix was skeletonized by ligation of the mesoappendix down to the appendix base at the ceco-appendiceal junction (Fig. 1B). A purse-string suture was then set along the border of the appendix base and the cecum, taking care that this suture penetrates all layers of the appendix including the mucosa. Great care was given to these two first steps, to securely disconnect the vascularization of the appendix. Then the intussusception of the appendix in the cecal lumen was performed using a blunt probe with a slightly smaller diameter than the diameter of the appendix at its tip (Fig. 1C). Care was taken not to 

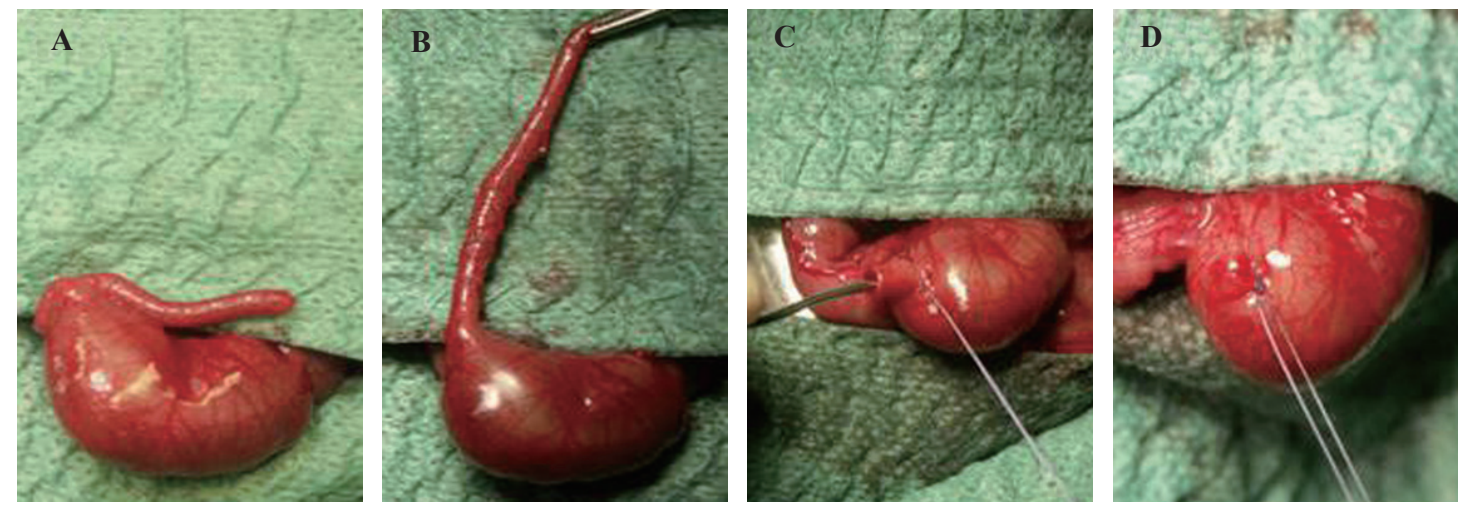

Fig. 1. Steps for the aseptic intussuscepted incidental appendectomy. A: cecum with appendix; B: completely skeletonized appendix; C: appendix intussusception; D: tied purse-string suture.

perforate the appendix. For easier handling, we held the appendix between two fingers with one hand. With the other hand we performed the inversion by pushing the probe from the appendix end in the direction of the cecum. The tip of the appendix with the probe in place should be felt between the fingers as the inversion of the appendix proceeds. This procedure was performed until the entire appendix was inside the cecum passing beyond the purse-string suture. The fold of the intussuscepted appendix was less than 2-3 $\mathrm{mm}$. The purse-string suture was tied (Fig. 1D) and a second purse-string suture was performed on the cecum in a distance of $2-3 \mathrm{~mm}$ displacing the nubbin in the cecal lumen.

After surgery, the procedure was explained to the ward nurses and the stools were controlled for appearance of the necrotic appendix. In one patient, autopsy with histological examination was performed.

\section{Results}

Two out of the three presented patients were neonates, who suffered from congenital diaphragmatic hernia where the colon, including the appendix, was in the thorax leaving a non-rotation status after reduction. One of the the two patients died from pulmonary complications on day 5 after diaphragmatic hernia repair. The third infant was 2 years old and had a large ovarian cyst to which the appendix was attached. The ovarian cyst was the reason for surgery.

In the two infants who survived, a small black slough was found in the stools on day seven and five after the operation. There was no sign of bleeding or intussusception in the two survivors during hospitalization.

In the child who died, autopsy showed a necrotic inverted appendix along with an intact cecal wall (Fig. 2). Histological examination confirmed that the inverted appendix was necrotic, and that some millimeters distally the cecal wall was intact with preserved vascularization.

Follow-up in the two survivors was 2 and 4 years postoperatively. They had no gastrointestinal disorders.

\section{Discussion}

Appendix removal, complementary to other abdominal surgery, intends to eliminate future appendicitis risk and to simplify the differential diagnoses in case of future abdominal pain. Since the majority of appendicitis cases occur during childhood, incidental appendectomy seems to be beneficial especially in pediatric patients. However, the question should be raised if this practice is still used today. Mulvihil et al ${ }^{[6]}$ reported a rate of $42 \%$ of incidental appendectomies out of 642 appendectomies. Using the known incidence of appendicitis, approximately 54 cases of acute appendicitis may have been obviated in the cohort referred to. ${ }^{[6]}$ However, according to the life expectancy of the reference population, the number of spared appendicitis cases might be overestimated.

Fisher et $\mathrm{al}^{[7]}$ as well as Snyder et a ${ }^{[8]}$ supported incidental appendectomy in patients below 35 years of age. In respect of costs, Sugimoto et al ${ }^{[9]}$ reported that between 1979 and 1981 the occurrence of incidental appendectomy exceeded that of appendicitis treated by appendectomy in South California. Since there is no actual study on the potential benefit from incidental appendectomy that applies to modern health systems, it is difficult to draw conclusions for today.

The fear of additional contamination is the main concern after performing incidental amputation appendectomy by transection of the appendix. It is undoubtedly true that some bacterial contamination occurs with transection of the appendix classifying the operation as a clean contaminated procedure 

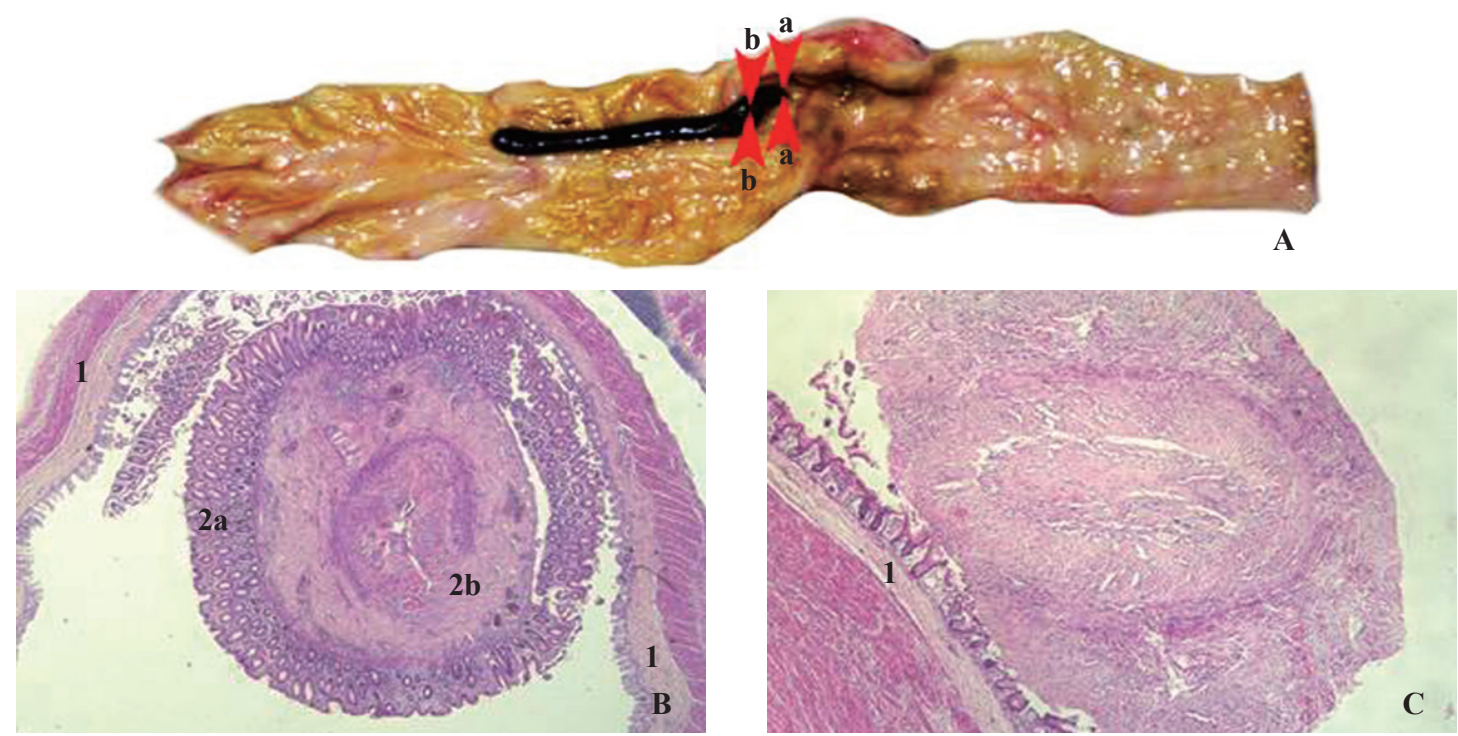

Fig. 2. Autopsy specimen. A: Opened cecum with inverted necrotic appendix and intact cecal wall: histological sections were taken at level a-a at the border between the inverted appendix and the cecum, and at level b-b some millimeters distally; B: Microscopic aspect at level (A-A) showing intact cecal wall (1), inverted mucosa of the appendix stump (2a), and appendix muscle wall (2b); C: Microscopic aspect at level (B-B) showing necrosis of the appendix, with merely distinguishable mucosa and muscle layer.

class 2 . In contrast surgery without opening the bowel like in inversion of the appendix is defined as a clean procedure class $1 .^{[10]}$ In addition to minimal contamination complementary appendectomy should diminish prolongation of operative time.

Inversion appendectomy could be considered a method satisfying the above criteria. Inversion appendectomy was described by Edebohls ${ }^{[11]}$ as early as 1895 and was "rediscovered" between the sixties and the eighties. ${ }^{[10,12,13]}$ A prospective randomized trial including 440 patients showed lower postoperative infection rate after inversion appendectomy as compared to conventional amputation appendectomy. ${ }^{[13]}$ Details on the method of inversion and additional ligation, similar to the AIIA technique we applied here, was described in 1991, where authors suggest a satisfying risk/benefit ratio. ${ }^{[14]}$

However in recent years, a number of complications were described after inversion appendectomy. It was in 1993 when the first publication on complications with intestinal hemorrhage 3 years after simple inversion appendectomy in a child after hepatic transplantation was reported, where the appendix devascularization was found to be incomplete. ${ }^{[4]}$ In 2001, the retention and inflammation of a simply inverted appendix 15 months after the operation in a neutropenic infant was described. ${ }^{[1]}$ After inversion and ligation procedure, an appendix was considered as a leading point for ileocecal intussusception on 6 days in an 18-month old child in $2008 .^{[2]}$

In 20 out of 41 adult patients, colonoscopy could reveal a persisting inverted appendix following simple inversion of the appendix. ${ }^{[3]}$ Since in these patients no symptoms were registered, the authors suggest that the persistence of the appendix is a welcome event carrying several benefits as the appendix continues to work as a specialized organ.

The division of the mesoappendix alone as in simple inversion of the appendix seems not to securely eliminate the entire blood supply of the appendix, which also comes from the cecal wall. Therefore, a proper suturing technique ensuring necrosis after inversion is necessary. We advocate dissection of the mesoappendix down to the base without impairment of the cecal wall, and a first purse-string suture to be performed directly at the fold of the inverted appendix taking all the layers including the mucosa, ensuring a complete strangulation of the appendix base. This technique is in contrast to the laparoscopic procedure where the base stump should be vascularized. With the second purse-string suture we performed, the appendix can definitively be pushed into the cecal lumen ensuring a complete closure of the cecal wall after necrosis of the appendix.

Appendix necrosis is confirmed if the appendix slough appears in the stools. It is important for the patient charts, especially in cases where later unclear abdominal complains occur, to record if the appendix slough passed. We experienced passage of the necrotic appendix in the two infants who survived. It might be discussed if colonoscopy should be performed in patients without passing of the appendix slough.

The major limitations of the present report are the 
retrospective design and the little number of patients reported. However, in this patient series, indication and surgical technique were standardized. We cannot support the point of view that inversion appendectomy causes additional morbidity if the indication is restricted to selected patients and if the technique applied completely interrupts the blood of all appendix wall layers as with the AIIA technique described above.

We conclude that in selected cases where postoperative misplacement of the appendix is predictable or where the appendix is indirectly involved in the main pathology, AIIA might be beneficial. We suggest prospective evaluation of this method. If applied, the surgical technique needs to result in complete devascularization of the appendix stump along with preservation of cecal vascularization. Stools should be screened in order to record if the appendix slough has passed.

Funding: None.

Ethical approval: Not needed.

Competing interest: None.

Contributors: All authors contributed to patient management, and data interpretation. All authors approved the final version of the manuscript.

\section{References}

1 McCarville MB, Ross MB, Rao BN, Furman WL. Sonographic appearance of appendicitis in a neutropenic pediatric patient after inversion appendectomy. Pediatr Radiol 2001;31:578-580.

2 Arora A, Caniano AC, Hammond S, Besner GE. Inversion appendectomy acting as a lead point for intussusception. Pediatr
Surg Int 2008;24:1261-1264.

3 Almasad JK, Daoud FS. Long-term outcome of simple inversion of the appendix as an alternative to incidental appendectomy. Ann Saudi Med 2008;28:179-182.

4 Reding R, Clapuyt P, Lengele B, Veyckemans F, Noel H, Sokal $\mathrm{E}$, et al. Intestinal hemorrhage three years after incidental appendectomy by total inversion. Eur J Pediatr Surg 1993;3:5960.

5 Wheeler RA, Malone PS. Use of the appendix in reconstructive surgery: A case against incidental appendicectomy. Br J Surg 1991;78:1283-1285.

6 Mulvihill S, Goldthorn J, Woolley MM. Incidental appendectomy in infants and children. Risk v rationale. Arch Surg 1983;118:714-716.

7 Fisher KS, Ross DS. Guidelines for therapeutic decision in incidental appendectomy. Surg Gynecol Obstet 1990;171:95-98.

8 Snyder TE, Selanders JR. Incidental appendectomy--yes or no? A retrospective case study and review of the literature. Infect Dis Obstet Gynecol 1998;6:30-37.

9 Sugimoto T, Edwards D. Incidence and costs of incidental appendectomy as a preventive measure. Am J Public Health 1987;77:471-475.

10 Lilly JR, Randolph JG. Total inversion of the appendix: experience with incidental appendectomy in children. J Pediatr Surg 1968;3:357-363.

11 Edebohls GM. Inversion of the vermiform appendix. Am J Med Sci 1895;109:650-656.

12 Bishop HC, Filston HC. An inversion-ligation technique for incidental appendectomy. J Pediatr Surg 1973;8:889-892.

13 Meissner K. Inversion versus amputation of the appendix: an objective comparison of 440 randomized cases (author's transl) Langenbecks Arch Chir 1980;353:129-138.

14 Voeller GR, Fabian TC. Inversion-ligation appendectomy for incidental appendectomy. Am J Surg 1991;161:483-484.

Received March 22, 2012 Accepted after revision May 18, 2012 